\title{
Influência da Interação Touro x Rebanho na Estimação da Correlação entre Efeitos Genéticos Direto e Materno em Bovinos da Raça Nelore
}

\author{
Joanir Pereira Eler ${ }^{1}$, José Bento Sterman Ferraz ${ }^{2}$, Bruce Lowel Golden ${ }^{3}$, Evandro Pereira ${ }^{4}$
}

RESUMO - A interação touro x rebanho foi avaliada em uma população com 30.789 registros de animais da raça Nelore nascidos entre 1984 e 1994 em doze fazendas localizadas em três Estados do Sudeste e Centro-Oeste brasileiro, com um total de 48.495 animais no pedigree. As características consideradas foram os pesos ao nascer (PESNAS) e à desmama (PESDES) e o ganho de peso da desmama ao sobreano (GP345). O efeito da interação touro x rebanho foi considerado aleatório em modelos animais uni e bicaraterística, usando MTDFREML. Esse efeito foi importante para PESNAS (6\% da variância fenotípica) e influenciou os componentes de variância e covariância e, conseqüentemente, os parâmetros genéticos. O efeito foi menor (cerca de 1\% da variância fenotípica) para PESDES, mas alterou as estimativas dos componentes de variância e covariância. Para GP345, o efeito foi pequeno, embora significativo pelos verossimilhança. As correlações genéticas entre efeitos direto e materno são próximas de zero, ou até mesmo positivas, se a interação touro x rebanho for incluída no modelo, e sempre negativas se ela for omitida.

Palavras-chave: correlação genética, efeitos direto e materno, modelo animal.

\section{Influence of Sire $x$ Herd Interaction on the Estimation of Correlation between Direct and Maternal Genetic Effects in Nellore Cattle}

\begin{abstract}
Sire $x$ herd interactions were studied in 30,789 records of birth (BW) and weaning weight (WW) and weight gain from weaning to 18 months of age (G345) of Nellore cattle born from 1984 to 1994 in twelve farms located in three states of central and southeastern Brazil, with a total of 48.495 animals in pedigree. Sire $\mathrm{x}$ herd interaction was considered as a random effect in single trait and two traits animal models using MTDFREML. This effect was important for BW ( $6 \%$ of the phenotypic variance) and it both affected variance and covariance components and, consequently, genetic parameters. The effect was smaller for WW (around $1 \%$ of the phenotypic variance), but influenced the estimates of (co) variance components. For G345, Sire x Herd effect was small. Likelihood tests showed that this effect was significant for all traits. This study showed that genetic correlations between direct $\mathrm{x}$ maternal effects are close to zero or even positive if sire $\mathrm{x}$ herd interaction is fitted in the model, and always negative if it is not.
\end{abstract}

Key Words: genetic correlation, direct and maternal effects, animal model

\section{Introdução}

O gado zebu é, hoje, criado nas diferentes regiões do Brasil em vários níveis de manejo. Determinado touro, por intermédio da inseminação artificial, pode produzir progênies em diferentes rebanhos em qualquer parte do país. O desenvolvimento da metodologia de avaliação genética por modelos mistos e a implementação do controle de desenvolvimento ponderal, por empresas privadas e também pela Associação Brasileira de Criadores de Zebu (ABCZ), em todo o território nacional, tornam possível a avaliação dos reprodutores dentro de grande amplitude de condições ambientais.
Há evidências da existência de interação genótipo $\mathrm{x}$ ambiente (principalmente touro $\mathrm{x}$ rebanho) nas raças zebuínas. A acurácia das estimativas de valor genético nos diferentes ambientes será reduzida se estas interações forem importantes. A menos que o touro tenha progênies em muitos ambientes, qualquer efeito da interação seria confundido com a estimativa do mérito genético. Isso limitaria tanto a capacidade de se estimarem as diferenças entre touros em condições específicas, como a efetividade da comparação de touros entre rebanhos (BUCHANAN e NIELSEN, 1979).

Alguns estudos têm sido conduzidos no sentido de examinar a importância das interações touro $x$ ambiente em gado de corte a campo. Esses estudos envolvem,

\footnotetext{
${ }^{1}$ Professor Associado da Fac. de Zootecnia e Engenharia de Alimentos, USP, Cx. Postal 23, 13630-970, Pirassununga, SP. E.mail: joapeler@usp.br

2 Professor Titular da Fac. de Zootecnia e Engenharia de Alimentos, USP. E.mail: jbferraz@usp.br

3 Professor Associado, Universidade do Estado do Colorado, EUA.

${ }^{4}$ Mestrando do Curso de Zootecnia (Qualidade e Produtividade Animal) da Fac. de Zootecnia e Engenharia de Alimentos, USP. E.mail: epereira-zoot@yahoo.com
} 
em geral, estimação de componentes de variância da interação, usando técnicas de análise de variância, podendo-se citar os de KENNEDY e HENDERSON (1975), estudando os efeitos da interação touro $\mathrm{x}$ ano de nascimento dos bezerros nas raças Hereford e Angus; NUNN et al.(1978); BUCHANAN e NIELSEN (1979), analisando os efeitos da interação touro x estação de nascimento do bezerro, na raça Maine-Anjou; e TESS et al.(1979), estudando a interação touro $x$ rebanho dentro de região.

Na maioria dos casos, o efeito da interação touro $\mathrm{x}$ região não tem sido significativo, enquanto o da interação touro $x$ rebanho tem sido consistentemente importante, principalmente no peso à desmama. TESS et al.(1979), por exemplo, encontraram, para o peso à desmama na raça Simental, variâncias para o componente de touro correspondentes a valores entre 0,2 e $1,6 \%$ da variância fenotípica total e para a interação touro x rebanho, valores entre 2,9 e 4,0. Assim, HENDERSON (1974) sugeriu a inclusão da interação touro $\mathrm{x}$ rebanho na predição dos valores genéticos dos animais.

A técnica de análise de variância não tem sido, no entanto, considerada a mais apropriada para estimação de componentes de variância em dados de campo (NOTTER et al., 1992), e a variação encontrada, aparentemente devida à interação touro x rebanho, poderia provir da heterogeneidade da variância residual e/ou da variância genética aditiva entre rebanhos (DICKERSON, 1962), do uso de pequeno número de touros selecionados, do acasalamento não-aleatório ou do tratamento preferencial de alguns grupos de meio-irmãos paternos.

BERTRAND et al. (1985), analisando dados da raça Hereford, nos quais o número de touros era relativamente grande (de 87 a 373), obtiveram componentes de variância da interação touro x rebanho igual ou mesmo maior que o componente entre touros. De qualquer forma, a importância da interação touro $\mathrm{x}$ rebanho tem sido vista com reserva à luz da sua menorimportância quando observada em experimentos delineados (MAHRT et al., 1990; NOTTER e CUNDIFF, 1991) e em análises de dados de campo usando diferente metodologia(GARRICKet al., 1989).

Entretanto, NOTTER et al. (1992), na raça Angus, na Austrália, usando o método de máxima verossimilhança restrita (REML), obtiveram estimativas significativas para o componente de variância da interação touro $x$ rebanho no peso à desmama. As estimativas corresponderam a valores entre 3,3 e $6,2 \%$ da variância fenotípica.
Nas raça Nelore, indicações da existência de interação touro $\mathrm{x}$ rebanho foram obtidas por ELER et al. (1989) e ELER et al. (1995), comparando-se, respectivamente, os resultados de análises, de um mesmo conjunto de dados, usando o método de quadrados mínimos e de máxima verossimilhança restrita (REML). Na primeira, os valores encontrados para o coeficiente de herdabilidade foram $0,42 \pm 0,03$; $0,24 \pm 0,02$ e $0,29 \pm 0,03$, respectivamente, para os pesos ao nascer, aos 205 e 365 dias de idade. Na segunda, os valores encontrados foram, respectivamente, $0,29 \pm 0,04 ; 0,14 \pm 0,02$ e $0,17 \pm 0,03$. Nenhuma das duas análises continha o efeito da interação no modelo, mas, na primeira, o efeito de touro foi aninhado dentro de rebanho (modelo hierárquico) e, neste caso, o componente de touro pode ter sido inflado pela interação touro x rebanho (TESS et al., 1979).

A importância da interação touro $x$ ambiente deve, portanto, ser considerada nos modelos de análise de modo a terem seus efeitos minimizados. Assim, os objetivos do presente trabalho foram estimar os componentes de (co)variância e os parâmetros genéticos para as características de desempenho ponderal, utilizando modelos matemáticos com e sem interação touro x rebanho; analisar, usando o método de máxima verossimilhança restrita, o conjunto de informações disponíveis da raça Nelore; e determinar a importância da interação touro $x$ rebanho e seu possível impacto nas estimativas de correlação entre efeitos genéticos direto e materno.

\section{Material e Métodos}

Os dados analisados pertencem à Agropecuária CFM Ltda, com sede em São José do Rio Preto, SP e os arquivos foram preparados pelo Grupo de Melhoramento Animal da Faculdade de Zootecnia e Engenharia de Alimentos da Universidade de São Paulo. Foram analisados dados de desenvolvimento ponderal de animais nascidos entre 1984 e 1994, em 12 fazendas, nos Estados de São Paulo, Mato Grosso do Sul e Goiás. Para este trabalho, analisaram-se as características peso ao nascer (PESNAS), peso à desmama (PESDES) e ganho de peso em 345 dias da desmama (GP345).

As fazendas referentes a este estudo pertencem a uma mesma empresa, de modo que os procedimentos básicos são padronizados. A estação de monta é curta (60 dias), ocorrendo, em geral, nos meses de novembro e dezembro. Nos últimos anos, passou a ser adotado um manejo inédito na raça Nelore, em que as novilhas são colocadas na estação de monta 
1644 Rev. bras. zootec.

aos 14 meses de idade. Para isto, foi adotada uma segunda estação de monta, também curta, nos meses de março e abril. Essa estação é apenas para as novilhas que entram na estação normal com 14 meses e não ficam prenhes. As novilhas participantes da segunda estação de monta que não emprenharem terão sua última chance na estação normal do ano seguinte, quando elas estarão com 24 meses.

Apesar de os procedimentos básicos serem padronizados, as propriedades estão localizadas em diferentes regiões, que vão do Noroeste de São Paulo, passando pelo Mato Grosso do Sul até Goiás. As variações de manejo, aliadas às diferenças climáticas e de qualidade de solo, podem gerar diferenças ambientais entre as fazendas, principalmente entre aquelas localizadas em Estados diferentes.

Os dados provenientes dessas fazendas foram editados, eliminando-se inicialmente os animais sem informações de pai e mãe, animais pertencentes a grupos de contemporâneos com apenas um touro e alguns possíveis animais com pesos fora da amplitude considerada normal.

Foram também definidos os "touros de conexão", isto é, touros com um número mínimo de cinco progênies em cada um de dois ou mais rebanhos.Grupos de contemporâneos que não possuíam pelo menos um "touro de conexão" foram eliminados. Finalmente, foram eliminadas as progênies de touros representados em apenas um grupo de contemporâneos e de touros com menos de cinco progênies no total. Após a edição final, o arquivo para análise constava de 30.789 registros, totalizando 48.495 animais na matriz de parentesco. As características da população analisada são apresentadas na Tabela 1.

Os grupos de contemporâneos foram formados por fazenda + ano de nascimento + grupo de manejo + sexo do animal. Os grupos de manejo (GM) referem-se aos lotes de animais que permaneceram juntos em determinada etapa da vida - por exemplo, durante a gestação (GM ao nascer), do nascimento até a desmama (GM à desmama) e da desmama ao sobreano (GM ao sobreano). Esses grupos foram identificados por números que não se repetem dentro de fazenda no mesmo ano. A inclusão dos grupos de manejo elimina a necessidade da inclusão da estação de monta, pois ambos se confundem.

Para estimação dos componentes de variância, foi utilizado o método de máxima verossimilhança restrita (REML) e um algoritmo livre de derivadas (DFREML), como descrito por SMITH e GRASER (1986), GRASER et al.(1987) e MEYER(1987).

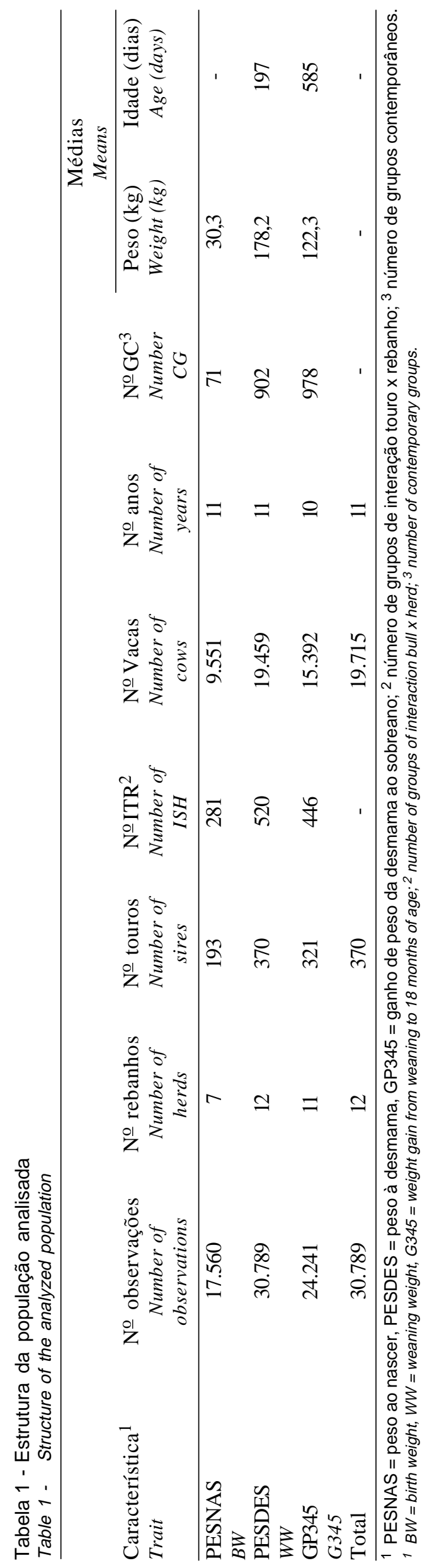


Utilizou-se como sofware básico o programa MTDFREML (multiple-trait derivative-free restricted maximum likelihood), desenvolvido por BOLDMAN e VAN VLECK (1991) e por BOLDMAN et al.(1993). Este programa utiliza o SPARSPAK (GEORGE et al., 1980), que permite aumentar a capacidade de trabalho dos computadores, em termos de memória disponível.

Para testar os efeitos da interação touro x rebanho, dois modelos matemáticos foram propostos, um contendo a interação e outro não. Em notação matricial, o modelo completo foi o seguinte:

$$
\mathrm{Y}=\mathrm{X} \beta+\mathrm{Zg}+\mathrm{Zm}+\mathrm{Zc}+\mathrm{Ztr}+\mathrm{e}
$$

em que $Y$ é vetor das variáveis dependentes: pesos ao nascer e à desmama e ganho de peso da desmama aos 18 meses de idade; $\mathbf{X}$, matriz de incidência dos efeitos fixos; $\boldsymbol{\beta}$, vetor dos efeitos fixos, incluindo grupo de contemporâneos (fazenda-ano-grupo de manejo e sexo), idade do animal à pesagem (dias) e idade da mãe ao parto (meses). Os dois últimos efeitos, incluídos como covariáveis; $\mathbf{Z}$, matriz de incidência dos efeitos aleatórios; $\mathbf{g}$, vetor dos efeitos aleatórios de valor genético aditivo direto do animal; $\mathbf{m}$, vetor dos efeitos aleatórios de valor genético aditivo materno do animal; c, vetor dos efeitos de ambiente permanente da vaca; tr, vetor dos efeitos da interação touro x rebanho; e, vetor dos erros aleatórios, e $\sim \operatorname{NID}\left(0, \sigma^{2}\right)$.

Os efeitos da interação genótipo $\mathrm{x}$ ambiente foram testados pela comparação das funções de verossimilhança do modelo completo e do modelo sem interação, utilizando-se o teste $\mathrm{C}^{2}$ (RAO, 1973; MOOD, 1973).

Os dados foram analisados de duas formas: a) considerando-se uma característica por vez (análise unicaracterítica) e b) cada uma das características em conjunto com o peso à desmama (análise bicaraterística).

Os efeitos genéticos maternos, os efeitos aleatórios de ambiente permanente da vaca e os efeitos fixos de idade da mãe ao parto não foram incluídos no modelo, para descrever o ganho de peso ao sobreano (GP345).

\section{Resultados e Discussão}

Os resultados obtidos, em ambas as análises uni e bicaraterística, encontram-se nas Tabelas 2 (componentes de variância e de covariância), 3 (parâmetros genéticos) e 4 (correlações).

Análise unicaracterística

Peso ao nascer

A contribuição do componente da interação touro $\mathrm{x}$ rebanho foi próxima de $6 \%$ da variância fenotípica. A inclusão desse componente no modelo influenciou

Tabela 2 - Componentes de variância e covariância obtidos nas análises unicaráter (UNI) e bicaráter (BI) em modelos sem (1) e com (2) interação touro $x$ rebanho

Table 2 - Variance and covariance components obtained in single-trait (ST) and two-trait (TT) analysis in models without (1) and with (2) interaction bull $x$ herd

\begin{tabular}{|c|c|c|c|c|c|c|c|c|c|}
\hline $\begin{array}{l}\text { Tipo } \\
\text { Type }\end{array}$ & $\begin{array}{c}\text { Modelo } \\
\text { Model }\end{array}$ & $\begin{array}{c}\text { Característica } \\
\text { Trait }\end{array}$ & $\begin{array}{c}{ }^{1} \sigma^{2} \mathrm{~g} \\
\left(\mathrm{~kg}^{2}\right)\end{array}$ & $\begin{array}{l}{ }^{2} \sigma^{2}{ }_{m} \\
\left(\mathrm{~kg}^{2}\right)\end{array}$ & $\begin{array}{l}{ }^{3} \sigma_{\mathrm{gm}} \\
\left(\mathrm{kg}^{2}\right)\end{array}$ & $\begin{array}{l}{ }^{4} \sigma^{2}{ }^{c} \\
\left(\mathrm{~kg}^{2}\right)\end{array}$ & $\begin{array}{l}{ }^{5} \sigma^{2}{ }_{\operatorname{tr}} \\
\left(\mathrm{kg}^{2}\right)\end{array}$ & $\begin{array}{l}{ }^{6} \sigma^{2} \mathrm{e} \\
\left(\mathrm{kg}^{2}\right)\end{array}$ & ${ }^{7}\left(\mathrm{~F}_{2}-\mathrm{F}_{1}\right)$ \\
\hline & 1 & $\operatorname{PESNAS}(B W)$ & 4,55 & 0,75 & $-0,61$ & 0,50 & - & 5,10 & $77^{* *}$ \\
\hline & 2 & $\operatorname{PESNAS}(B W)$ & 2,60 & 0,29 & 0,34 & 0,47 & 0,59 & 5,98 & \\
\hline UNI & 1 & $\operatorname{PESDES}(W W)$ & 88,39 & 20,67 & $-10,21$ & 33,37 & - & 186,5 & $27^{* *}$ \\
\hline \multirow[t]{7}{*}{$S T$} & 2 & $\operatorname{PESDES}(W W)$ & 72,88 & 18,33 & $-3,11$ & 32,38 & 4,49 & 193,2 & \\
\hline & 1 & GP345 (G345) & 67,51 & - & - & - & - & 222,3 & $19^{* *}$ \\
\hline & 2 & GP345 (G345) & 62,61 & - & - & - & 3,66 & 224,5 & \\
\hline & 1 & PESNAS + PESDES $(B W+W W)$ & 3,96 & 0,90 & $-0,46$ & 0,30 & - & 5,37 & $98^{* *}$ \\
\hline & 2 & PESNAS + PESDES $(B W+W W)$ & 2,66 & 0,62 & 0,16 & 0,28 & 0,38 & 5,94 & \\
\hline & 1 & PESDES + PESNAS $(W W+B W)$ & 88,91 & 19,93 & $-5,77$ & 30,01 & - & 187,0 & $98^{* *}$ \\
\hline & 2 & PESDES + PESNAS $(W W+B W)$ & 79,26 & 23,32 & $-4,08$ & 27,57 & 3,81 & 190,4 & \\
\hline BI & 1 & PESDES + GP345 $(W W+G 345)$ & 86,16 & 21,28 & $-9,94$ & 33,12 & - & 187,6 & $48^{* *}$ \\
\hline \multirow[t]{3}{*}{$T T$} & 2 & PESDES + GP345 $(W W+G 345)$ & 70,24 & 18,99 & $-2,65$ & 31,99 & 4,82 & 194,4 & \\
\hline & 1 & GP345 + PESDES $(G 345+W W)$ & 66,67 & - & - & - & - & 222,9 & $47^{* *}$ \\
\hline & 2 & GP345 + PESDES $(G 345+W W)$ & 61,88 & - & - & - & 3,52 & 225,1 & \\
\hline
\end{tabular}

${ }^{1} \sigma^{2} g=$ variância dos efeitos genéticos aditivos diretos; ${ }^{2} \sigma^{2}{ }_{m}=$ variância dos efeitos genéticos aditivos maternos; ${ }^{3} \sigma_{g m}=$ covariância entre efeitos genéticos aditivos direto e materno; ${ }^{4} \sigma^{2}{ }_{c}=$ variância dos efeitos de ambiente permanente da vaca; ${ }^{5} \sigma^{2}{ }_{\text {tr }}=$ variância da interação touro $\mathrm{x}$ rebanho; ${ }^{6} \mathrm{\sigma}^{2}{ }_{\mathrm{e}}=$ variância dos efeitos residuais; ${ }^{7}$ diferença entre os valores das funções de verossimilhança para os modelos com (F2) e sem interação (F1).

${ }^{1} \sigma^{2}{ }_{g}=$ direct additive genetic variance; ${ }^{2} \sigma_{m}^{2}=$ maternal additive genetic variance; ${ }^{3} \sigma_{g m}=$ covariance between direct additive and maternal additive genetic effects; ${ }^{4} \sigma_{c}^{2}=$ permanentenvironmental variance; ${ }^{5} \sigma_{t r}^{2}=$ variance of the interaction bull $x$ herd; ${ }^{6} \sigma^{2}{ }_{e}=$ environmental variance; ${ }^{7}$ difference between the values of likelihood functions for models with (F2) and without interaction (F1). 
1646 Rev. bras. zootec.

Tabela 3 - Estimativas de parâmetros genéticos obtidas nas análises unicaracterística (UNI) e bicaracterística (BI) em modelos sem (1) e com (2) interação touro x rebanho

Table 3 - Estimates of genetic parameters obtained in single-trait (ST) and two-trait (TT) analysis in models without (1) and with (2) interaction bull $x$ herd

\begin{tabular}{|c|c|c|c|c|c|c|c|c|c|}
\hline $\begin{array}{l}\text { Tipo } \\
\text { Type }\end{array}$ & $\begin{array}{c}\text { Modelo } \\
\text { Model }\end{array}$ & $\begin{array}{c}\text { Característica } \\
\text { Trait } \\
\end{array}$ & ${ }^{1} \mathrm{~N}$ & ${ }^{2} h^{2} g$ & ${ }^{3} \mathrm{~h}^{2} \mathrm{~m}$ & ${ }^{4} r_{g m}$ & $5 c^{2}$ & $6_{\mathrm{tr}^{2}}$ & $7 \mathrm{e}^{2}$ \\
\hline & 1 & $\operatorname{PESNAS}(B W)$ & 17.560 & 0,44 & 0,07 & $-0,33$ & 0,05 & - & 0,50 \\
\hline & 2 & $\operatorname{PESNAS}(B W)$ & 17.560 & 0,25 & 0,03 & 0,39 & 0,05 & 0,06 & 0,58 \\
\hline UNI & 1 & $\operatorname{PESDES}(W W)$ & 30.789 & 0,28 & 0,06 & $-0,24$ & 0,10 & - & 0,53 \\
\hline \multirow[t]{7}{*}{$S T$} & 2 & $\operatorname{PESDES}(W W)$ & 30.789 & 0,23 & 0,06 & $-0,09$ & 0,10 & 0,01 & 0,61 \\
\hline & 1 & GP345 (G345) & 24.241 & 0,23 & - & - & - & - & 0,77 \\
\hline & 2 & GP345 (G345) & 24.241 & 0,22 & - & - & - & 0,01 & 0,77 \\
\hline & 1 & PESNAS + PESDES $B W+W W$ & 17.560 & 0,39 & 0,09 & $-0,24$ & 0,03 & - & 0,53 \\
\hline & 2 & PESNAS + PESDES $B W+W W$ & 17.560 & 0,26 & 0,06 & 0,13 & 0,03 & 0,04 & 0,59 \\
\hline & 1 & PESDES + PESNAS $W W+B W$ & 30.789 & 0,28 & 0,06 & $-0,14$ & 0,09 & - & 0,58 \\
\hline & 2 & PESDES + PESNAS $W W+B W$ & 30.789 & 0,25 & 0,07 & $-0,09$ & 0,09 & 0,02 & 0,59 \\
\hline BI & 1 & PESDES + GP345 $W W+G 345$ & 30.789 & 0,27 & 0,07 & $-0,23$ & 0,10 & - & 0,59 \\
\hline \multirow[t]{3}{*}{$T T$} & 2 & PESDES + GP345 $W W+G 345$ & 30.789 & 0,22 & 0,06 & $-0,07$ & 0,10 & 0,01 & 0,61 \\
\hline & 1 & GP345 + PESDES $G 345+W W$ & 24.241 & 0,23 & - & - & - & - & 0,77 \\
\hline & 2 & GP345 + PESDES $G 345+W W$ & 24.241 & 0,21 & - & - & - & 0,01 & 0,77 \\
\hline
\end{tabular}

${ }^{1} \mathrm{~N}=$ número de observações; ${ }^{2} \mathrm{~h}^{2} \mathrm{~g}=$ coeficiente de herdabilidade para efeitos genéticos aditivos diretos; ${ }^{3} \mathrm{~h}^{2} \mathrm{~m}=$ coeficiente de herdabilidade para efeitos genéticos aditivos maternos; ${ }^{4} \mathrm{r}_{\mathrm{gm}}=$ correlação genética entre efeitos genéticos aditivos direto e materno; ${ }^{5} \mathrm{c}^{2}=$ proporção da variância fenotípica devida ao ambiente permanantes da vaca; $\operatorname{tr}^{2}=$ proporção da variância fenotípica devida à interação touro x rebanho; $7 \mathrm{e}^{2}=$ proporção da variância fenotípica devida aos efeitos residuais.

${ }^{1} \mathrm{~N}=$ number of observations; ${ }^{2} h^{2}=$ coefficient of heritability for direct additive genetic effects; ${ }^{3} h^{2}{ }_{m}=$ coefficient of heritability for maternal additive genetic effects ${ }^{4} r_{a m}=$ genetic correlation between direct and maternal addictive genetic effects; ${ }^{5} c^{2}=$ proportion of the phenotypic variance due to permanent environment; ${ }_{t t^{2}}{ }^{2}=$ proportion of the phenotypic variance due to the interaction bull $x$ herd; ${ }^{7} e^{2}=$ proportion of the phenotypic variance due to residual effects.

de forma expressiva a estimação dos componentes de variância dos efeitos genéticos direto e materno e da covariância entre efeitos genéticos direto e materno (Tabela 2). Para peso ao nascer, o valor da herdabilidade para efeito genético direto $\left(\mathrm{h}_{\mathrm{g}}^{2}\right)$ foi igual a 0,44 no modelo 1 (sem interação) e 0,25 no modelo 2 (com interação). O mesmo comportamento pode ser observado para o componente materno $\left(\mathrm{h}^{2}{ }_{\mathrm{m}}\right)$. O valor de $\mathrm{h}_{\mathrm{m}}^{2}$ sofreu redução de 0,07 (modelo 1 ) para 0,03 (modelo 2). A correlação entre efeitos genéticos direto e materno $\left(\mathrm{r}_{\mathrm{gm}}\right)$ foi o componente mais influenciado, mudando de $-0,33$ (modelo 1 ) para $+0,39$ (modelo 2 ).

\section{Peso à desmama}

Apesar de a contribuição da interação ter sido de, aproximadamente, $1,5 \%$ da variância fenotípica, foi suficiente para reduzir $\mathrm{h}_{\mathrm{g}}^{2}$ de 0,28 para 0,23 . O valor de $\mathrm{h}^{2}{ }_{\mathrm{m}}$ não se alterou, mas $\mathrm{r}_{\mathrm{gm}}$ elevou-se de -0,24 para-0,09. Ganho de peso da desmama ao sobreano (GP345)

Análises preliminares já haviam indicado que os efeitos maternos e de ambiente permanente da vaca foram desprezíveis nesta característica. Assim, no modelo 1, incluiu-se apenas o efeito genético direto e no modelo 2 , os efeitos genético direto e o efeito da interação touro x rebanho. A contribuição da interação para a variância fenotípica foi de $1,3 \%$ e a alteração de $\mathrm{h}^{2}$ g foi muito pequena (de 0,23 para 0,22 ).

\section{Análise bicaracterística}

Por esta análise, além da estimação dos componentes de variância (Tabela 2) e dos parâmetros genéticos (Tabela 3), parcialmente corrigidos para os efeitos de seleção nos dados, foram obtidas também as correlações entre as características (Tabela 4).

Peso ao nascer

$\mathrm{O}$ componente da interação touro $\mathrm{x}$ rebanho contribuiu com $4 \%$ da variância fenotípica. Os valores obtidos para $\mathrm{h}_{\mathrm{g}}{ }_{\mathrm{g}}$ foram 0,39 e 0,26 para os modelos 1 e 2 , respectivamente. Houve redução do valor de $\mathrm{h}^{2}{ }_{\mathrm{m}}$ de 0,09 (modelo 1) para 0,06 (modelo 2). O valor de $\mathrm{r}_{\mathrm{gm}}$ passou de $-0,24$ (modelo 1) para $+0,13$ (modelo 2 ). Peso à desmama

A contribuição da interação foi de aproximadamente $2 \%$, reduzindo $h^{2}{ }_{g}$ de 0,28 para 0,25 , na análise que incluiu PESDES + PESNAS, e de 0,27 para 0,22 , na que incluiu PESDES + GP345. Não houve alteração significativa de $\mathrm{h}^{2}{ }_{\mathrm{m}}$. Em relação ao valor de $\mathrm{r}_{\mathrm{gm}}$, a redução (em módulo) foi maior na análise que incluiu o GP345 (de -0,23 para -0,07 para os modelos 
ELER et al.

Tabela 4 - Coeficientes de herdabilidade dos efeitos genéticos direto e materno (diagonal) e correlação genética entre as características (abaixo da diagonal)

Table 4 - Coefficients of heritability of direct and maternal genetic effects (diagonal) and genetic correlation between the traits (below diagonal)

\begin{tabular}{|c|c|c|c|c|c|c|}
\hline & $\begin{array}{c}\text { Característica } \\
\text { Trait }\end{array}$ & $\begin{array}{c}\text { PESNAS }{ }^{1} \mathrm{D} \\
B W^{1} D\end{array}$ & $\begin{array}{c}\text { PESNAS }{ }^{2} \mathrm{M} \\
B W{ }^{2} M\end{array}$ & $\begin{array}{c}\text { PESDESD } \\
W W D\end{array}$ & $\begin{array}{c}\text { PESDESM } \\
W W M\end{array}$ & $\begin{array}{c}\text { GP345D } \\
G 345 D\end{array}$ \\
\hline Modelo & PESNAS D $(B W D)$ & 0,39 & - & - & - & - \\
\hline sem & PESNAS M $(B W M)$ & $-0,24$ & 0,09 & - & - & - \\
\hline interação & PESDES D $(W W D)$ & 0,64 & 0,26 & $0,28(0,27)$ & - & - \\
\hline Model without & PESDES M $(W W M)$ & $-0,59$ & 0,62 & $-0,14(-0,23)$ & 0,06 & - \\
\hline interaction & GP345 D $(G 345 D)$ & - & - & 0,20 & $-0,08$ & 0,23 \\
\hline Modelo & PESNAS D $(B W D)$ & 0,26 & - & - & - & - \\
\hline com & PESNAS M $(B W M)$ & 0,13 & 0,06 & - & - & - \\
\hline interação & $\operatorname{PESDES} \mathrm{D}(W W D)$ & 0,77 & 0,37 & $0,25(0,26)$ & - & - \\
\hline Model with & PESDES M $(W W M)$ & $-0,60$ & 0,67 & $-0,09(-0,07)$ & 0,07 & - \\
\hline interaction & GP345 D (G345 D) & - & - & 0,26 & $-0,12$ & 0,21 \\
\hline
\end{tabular}

${ }^{1} \mathrm{D}=$ efeito direto; ${ }^{2} \mathrm{M}=$ efeito materno.

${ }^{1} D=$ direct effect; ${ }^{2} M=$ maternal effect.

1 e 2 , respectivamente) em relação àquela que incluiu o PESNAS (de -0,14 para -0,09).

\section{GP345}

O modelo 1, da mesma forma que na análise unicaracterística, incluiu apenas o efeito genético direto, além do residual. O modelo 2 incluíu também a interação touro $\mathrm{x}$ rebanho. A contribuição da interação foi pouco mais de $1 \%$. O valor de $\mathrm{h}^{2}{ }_{\mathrm{g}}$ mudou de 0,23 para 0,21 , alteração pequena do ponto de vista biológico.

Embora a contribuição da interação tenha sido pequena em termos de percentual da variância fenotípica, seus efeitos foram significativos $(\mathrm{P}<0,01)$ para as três características analisadas, segundo o teste de qui-quadrado para a razão de máxima verossimilhança (RAO, 1973; MOOD, 1973). Deve ser considerado que este estudo envolveu apenas fazendas de uma mesma empresa e, portanto, com certa similaridade, principalmente no manejo. As diferenças entre as fazendas serão indicutivelmente maiores, se forem considerados os rebanhos dos diversos Estados brasileiros.

As alterações dos componentes genéticos direto e materno foram substanciais nos pesos ao nascer e à desmama. Todavia, o efeito mais importante da interação touro $\mathrm{x}$ rebanho foi sobre a correlação entre os efeitos genéticos direto e materno $\left(\mathrm{r}_{\mathrm{gm}}\right)$, com valores passando de negativo a positivo, ou se aproximando de zero, conforme o modelo de 1 (sem interação) para 2 (com interação touro x rebanho).
LEE e POLLAK (1996) obtiveram mudanças similares, quando consideraram a interação touro $\mathrm{x}$ ano de nascimento do bezerro, tanto em análises de dados de simulação quanto em dados reais na raça Simental.

Nas análises para predição do valor genético em gado de corte, normalmente assumem-se os efeitos genéticos direto e materno como correlacionados e não se inclui a interação genótipo $\mathrm{x}$ ambiente no modelo. Dessa forma, a covariância, em geral negativa, pode estar inflada em sua magnitude pela interação, forçando os animais com DEPs positivas para peso a apresentarem DEPs negativas para habilidade materna e vice-versa, ou seja, uma ordenação incorreta dos animais.

Considerando que a correlação negativa entre os efeitos genéticos direto e materno pode ser resultado de estimação incorreta, MEYER (1991) sugere que esses efeitos sejam assumidos como não-correlacionados. GOLDEN $^{1}$ (1995) também assume esta covariância como zero, na predição de valores genéticos.

$\mathrm{O}$ aumento da capacidade de memória e da velocidade dos computadores atuais tornou possível a inclusão da interação genótipo $\mathrm{x}$ ambiente nos modelos de análise genética em gado de corte; assim, os efeitos genéticos direto e materno poderiam ser assumidos como correlacionados. Alguns estudos são ainda necessários, inclusive, para definir a melhor forma de incluir os efeitos da interação, seja como touro $\mathrm{x}$ rebanho, touro $\mathrm{x}$ ano ou touro $\mathrm{x}$ grupo contemporâneo.

\footnotetext{
${ }^{1}$ GOLDEN, B.L. South American BLUP Workshop. Fort Collins, Colorado, julho de 1995. (Comunicação pessoal)
} 


\section{Conclusões}

A contribuição da interação touro $\mathrm{x}$ rebanho obtida neste trabalho foi pequena, mas suficiente para alterar a magnitude e o sinal da correlação entre efeitos genéticos direto e materno.

A inclusão da interação genótipo $x$ ambiente, seja touro $\mathrm{x}$ rebanho, touro $\mathrm{x}$ grupo contemporâneo ou touro $\mathrm{x}$ ano de nascimento do bezerro, no modelo, pode ser um procedimento adequado para melhorar a ordenação dos reprodutores.

\section{Agradecimento}

À Agropecuária CFM, pela cessão dos dados, ao CNPq e à FAPESP, pelo apoio financeiro.

À Tecnóloga em Processamento de Dados, Elisângela Chicaroni de Mattos Oliveira, pelo auxílio no processamento dos arquivos.

\section{Referência Bibliográficas}

BERTRAND, J.K., BERGER, P.J., WILLHAM, R.L. 1985. Sire x Environment interactions in beef cattle weaning weight field data. J. Anim. Sci., 60(6):1396-1402.

BERTRAND, J.K., HOUGH, J.D., BENYSHEK, L.L. 1987. Sire x Environment interactions and genetic correlations of sire progeny performance across regions in dam-adjusted field data. J. Anim. Sci., 64(1):77-82.

BOLDMAN, K.G., KRIESE, L.A., VAN VLECK, L.D. et al. 1993. A manual for use of MTDFREML: a set of programs to obtain estimates of variances and covariances. USDA-ARS. 120p.

BOLDMAN, K.G., VAN VLECK, L.D. 1991. Dervative-free restricted maximum likelihood estimation in animal models with sparse matrix solver. J. Dairy Sci., 74(12):4337- 4343.

BUCHANAN, D.S., NIELSEN, M.K. 1979. Sire x environment interactions in beef cattle field data. J. Anim. Sci., 48(2):307-312.

BURFENING, P.J., KRESS, D.D., FRIEDRICH, R.L. et al. 1978. Calving ease and growth rate of Simental sired calves. II. Genetic parameter estimates. J. Anim. Sci., 46(4):930-936.

DICKERSON, G.E. 1962. Implications of genetic-environment interaction in animal breeding. Anim. Prod., 4:47-55.

ELER, J.P. Utilização de modelos animais univariado e multivariado na avaliação genética de bovinos. Pirassununga, SP: FZEA, 1994. 123p. Tese (Livre Docência) - Faculdade de Zootecnia e Engenharia de Alimentos/Universidade de Sào Paulo, 1994.

ELER, J.P., LÔBO, R.B., ROSA, A.N. 1989. Influência de fatores genéticos e de meio em pesos de bovinos da raça Nelore criados no Estado de São Paulo. R. Soc. Bras. Zootec., 18(2): 103-111.

ELER, J.P., VAN VLECK, L.D., FERRAZ, J.B.S. et al. 1995. Estimation of variances due to direct and maternal effects for growth traits of Nelore cattle. J. Anim. Sci., 73(11): 3253-3258.

GARRICK, D.J., POLAK, E.J., QUAAS, R.L. 1989. Variance heterogeneity in direct and maternal weight traits by sex and percent purebred for Simental sired calves. J. Anim. Sci., 67(10):2515-2528

GEORGE, A., LIU, J., NG, E. 1980. User guide for SPARSPACK: Waterloo sparse linear equations package. Canada: Dept. Computer Sci. - Univ. Waterloo.
GRASER, H.U., SMITH, S.P., TIER, B. 1987. A Derivative-free approach for estimating variance components in animal models by restricted maximum likelihood. J. Anim. Sci., 64(5):1362-1370.

HARVILLE,D.A. 1977. Maximum likelihood approaches to variance component estimation and to related problems. J. Am. Stat. Assoc., 72(358):320-340.

HENDERSON, C.R. 1950. Estimation of genetic parameters (Abstract). Ann. Math. Stat., 21:309-310.

HENDERSON, C.R. 1963. Selection index and expected genetic advance. In: HANSON, W.D., ROBINSON, H.F. (Eds.) Statistical genetics and plant breeding. Washington, D.C.: NAS-NRC982. p.141-163.

HENDERSON,C.R. 1974. General flexibility of linearmodel techniques for sire evaluation. J. Dairy Sci., 57:963-981.

HENDERSON, C.R. 1975. Best linear unbiased estimation and prediction under a selection model. Biometrics. 31(2):423-447.

KENNEDY, B.W., HENDERSON, C.R. 1975. Components of variance of growth traits among Hereford and Aberdeen Angus calves. Can. J. Anim. Sci., 55(4):493-502.

LEE, C., POLLAK, E.J. 1996. Comparison of sire-by-yearinteraction model to a model including genetic correlation between direct and maternal effects. J. Anim. Sci., 73(suppl.1):116.

MAHRT, G.S., NOTTER, D.R., BEAL, W.E. et al. 1990. Growth of crossbred progeny of polled Hereford sires divergently selected for yearling weight and maternal ability.J. Anim. Sci., 68(7):18891898.

MEYER, K. 1987. Estimates of variances due to sire $x$ herd interactions and environmental covariances between paternal half-sibs for first lactation dairy production. Livest. Prod. Sci., 17(2): 95-115.

MEYER, K. 1989. Resticted maximum likelihood to estimate variance components for animal models with several random effects using a derivative-free algorithm. Genet. Sel. Evol., 21(3): 317-340.

MEYER, K. 1991. Estimating variances and covariances formultivariate animal models by restricted maximum likelihood. Genet. Sel. Evol., 23(1): 67-83.

MOOD, A.M., GRAYBILL, F.A., BOES, D.C. 1973. Introduction to the theoryof statistics. 3.ed. New York: McGraw-HillPublishing Co. $564 \mathrm{p}$.

NELDER, J.A., MEAD, R. 1965. A Simplex method for function maximization. Computer J., 5:147-151.

NOTTER, D.R., CUNDIFF, L.V. 1991. Across-breed expected progeny differences: use of within-breed expected progeny differences to adjust breed evaluations for sire sampling and genetic trend. J. Anim. Sci., 69(12):4763-4776.

NOTTER, D.R., TIER, B., MEYER, K. 1992. Sire x Herd interactions for weaning weight in beef cattle. J. Anim. Sci., 70(8): 2359-2365. 1.

NUNN, T.R., KRESS, D.D., BURFENING, P.J. et al. 1978. Region by sire interaction for reproduction traits in beef cattle. J. Anim. Sci., 46(4):957-964.

RAO, C.R. 1973. Linear statistical inference and its applications. 2.ed. New York: J.Willey and Sons. p.417-420.

SEARLE, S.R. 1992. Notes on variance component estimation. New York: Cornel University. 146p.

SMITH, S.P., GRASER, H.U. 1986. Estimating variance components in a class of mixed models by restricted maximum likelihood. J. Dairy Sci., 69(4):1156-1165.

TESS, M.W., KRESS, D.D., BURFENING, P.J. et al. 1979. Sire x Environment interactions in Simental-sired calves. J. Anim. Sci., 49(4):964-971.

Recebido em: $16 / 12 / 98$

Aceito em: 24/04/00 